\title{
Laparoscopic living donor hepatectomy
}

\author{
Ki-Hun Kim, Young-Dong Yu, Dong-Hwan Jung, Tae-Yong Ha, \\ Gi-Won Song, Gil-Chun Park, Shin Hwang, and Sung-Gyu Lee
}

\begin{abstract}
Division of Hepatobiliary Surgery and Liver Transplantation, Department of Surgery, University of Ulsan College of Medicine and Asan Medical Center, Seoul, Korea
\end{abstract}

\begin{abstract}
Shortage of deceased donor organs led to establishment of living donor liver transplantation. Recent reports have strongly suggested that laparoscopic approach should be the gold standard for lesions in the left lateral section. Laparoscopic living donor left lateral sectionectomy was first described in 2002. Subsequently, laparoscopic procurement of left lateral sections was shown to be safe and reproducible, resulting in grafts similar to those obtained with open surgery. In 2006, laparoscopy-assisted right lobe donor hepatectomy was reported. To date, however, only a small number of liver transplant centers have performed laparoscopic donor hepatectomy because the procedure can be performed only by surgical teams with extensive expertise in performing both minimally invasive surgery on the liver and liver transplantation with partial and living donor liver grafts. Herein, we describe the details of laparoscopic living donor hepatectomy including total laparoscopic surgery and laparoscopy-assisted surgery. (Korean J Hepatobiliary Pancreat Surg 2012;16:47-54)
\end{abstract}

Key Words: Laparoscopic surgery; Laparoscopy-assisted surgery; Donor hepatectomy; Living donor liver transplantation

\section{INTRODUCTION}

Due to the shortage of deceased donor organs, living donor liver transplantation (LDLT) has become an established treatment modality for patients with acute and chronic liver disease. The first successful pediatric LDLT, using a left lateral section graft from a mother to her son, was performed in Brisbane, Australia in 1989. Thereafter this life-saving procedure has been applied to adult patients.

Recent reports have strongly suggested that laparoscopic approach can be the gold standard for lesions in the left lateral section. Laparoscopic living donor left lateral sectionectomy was first described in 2002, indicating that this technique was feasible for pediatric LDLT. Subsequently, laparoscopic procurement of left lateral sections was shown to be safe and reproducible, resulting in grafts similar to those obtained with open surgery.

In 2006, laparoscopy-assisted right lobe donor hepatectomy was reported. Laparoscopic donor nephrectomy has become the standard method of procuring kidneys for living donor renal transplantation because it is associated with lower rates of donor morbidity without having any deleterious effects on long-term renal function in recipients. To date, however, only a small number of centers have performed laparoscopic donor hepatectomy because the procedure can be performed only by surgical teams with extensive expertise in performing both minimally invasive surgery on the liver and liver transplantation with partial and living donor liver grafts. ${ }^{1-8}$

Herein, we describe the details of laparoscopic living donor hepatectomy including total laparoscopic surgery and laparoscopy-assisted surgery.

\section{TOTAL LAPAROSCOPIC LIVING DONOR HEPATECTOMY}

Laparoscopic living donor left lateral sectionectomy in pediatric LDLT is introduced because total laparoscopic living donor right hepatectomy is not clearly reported un-

Received: May 1, 2012; Revised: May 10, 2012; Accepted: May 21, 2012

Corresponding author: Ki-Hun Kim

Division of Hepatobiliary Surgery and Liver Transplantation, Department of Surgery, University of Ulsan College of Medicine and Asan Medical Center, 388-1, Poongnap-dong, Songpa-gu, Seoul 138-736, Korea

Tel: +82-2-3010-3495, Fax: +82-2-3010-6701, E-mail: khkim620@amc.seoul.kr 


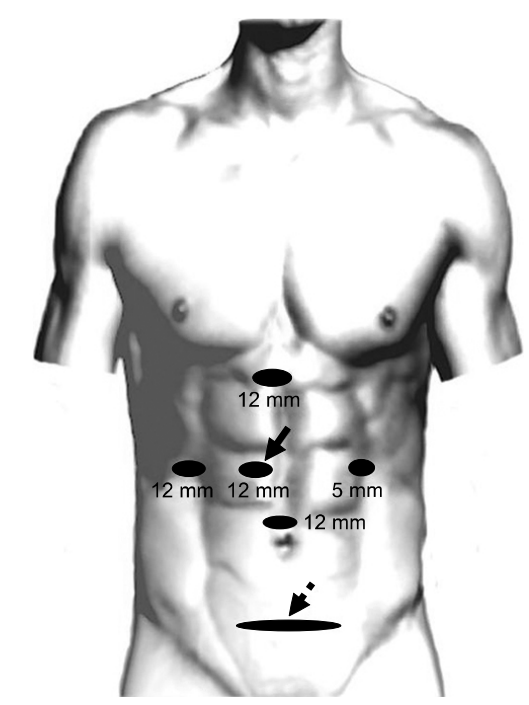

Fig. 1. Trocar position: A solid arrow indicates a main working port of CUSA and a dotted arrow indicates a graft retrieval site.

til now.

\section{Laparoscopic living donor left lateral sectio- nectomy}

The donor was placed supine in the $30^{\circ}$ reversed Trendelenburg position, with the surgeon standing between the donor's legs. No Pringle maneuver was used during parenchyma division. Carbon dioxide pressure to the pneumoperitoneum was maintained at $12 \mathrm{mmHg}$. One monitor each was placed on the middle and right side of the donor. Five trocars were usually inserted, with the middle trocar used as the primary working device (Fig. 1).

The liver was inspected with a $30^{\circ}$ laparoscope and partially resected for a frozen biopsy to confirm the degree of steatosis. The left triangular and falciform ligament were divided with a harmonic scalpel (Ultracision, Ethicon Endosurgery, Cincinnati, $\mathrm{OH}$ ) to free the left lateral sector and the round ligament, used as a supporter, was divided after complete dissection of the left side hepatoduodenal ligament. The left hepatic artery and portal vein were identified and taped using an atraumatic grasper (Direct Drive Laparoscopic Grasper, Applied Medical Resources, Rancho Santa Margarita, CA, US) and monopolar dissector. Some small branches going to the caudate lobe were clipped and divided to get sufficient lengths of the left hepatic artery and portal vein. The deep hepatic

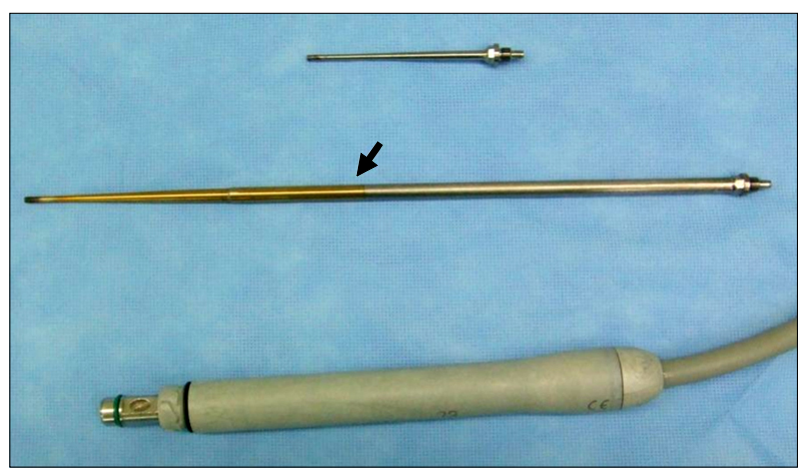

Fig. 2. An arrow indicates a laparoscopic ultrasonic aspirator with a long tip.

parenchyma were divided along the right side of the round and falciform ligament, using a laparoscopic ultrasonic aspirator (CUSA Exel, Valleylab corp., CO, US) with a long tip (Fig. 2). However, the liver capsule and superficial parenchyma were dissected using a harmonic scalpel. The glissonian pedicles of segment 4 were ligated using a knot pusher (Knot Guide $5 \mathrm{~mm}$, MGB Endoscopy corp., Seoul, Korea) or were clipped and divided for hemostasis and biliostasis. When the liver division reached the left hepatic vein, the left lateral sector was surrounded by a cotton tape which was passed under the left hepatic vein, portal vein and hepatic artery for a liver "hanging-over" maneuver. The left bile duct was exposed after complete division of the remnant hepatic parenchyma and was cut just above the Hem-O-Lock clip (Weck Closure System, Research Triangle Park, NC, US), which was clipped onto the proposed target level of the left hepatic duct.

After the recipient was ready to receive the graft, a 10 $\mathrm{cm}$-sized suprapubic incision was made and a $12 \mathrm{~mm}$ trocar was inserted for procurement. After infusion of 5000 $\mathrm{U}$ heparin, the proximal end of the left hepatic artery was clipped and divided using a Hem-O-Lock clip, and both ends of left portal vein were clipped and divided. A unilateral linear stapler (Endo TA $30 \mathrm{~mm}$, US Surgical, Norwalk, CT, US) was used to cut the left hepatic vein. The graft was placed into the endo-bag and retrieved through the suprapubic incision site.

The graft was flushed on the back table with $1 \mathrm{~L}$ of $4^{\circ} \mathrm{C}$ HTK solution (Odyssey Pharmaceuticals, East Hanover, NJ, US) through the left portal vein. The suprapubic wound was then closed and $\mathrm{CO}_{2}$ gas was re-insufflated to check the control of hemostasis and biliostasis on the 

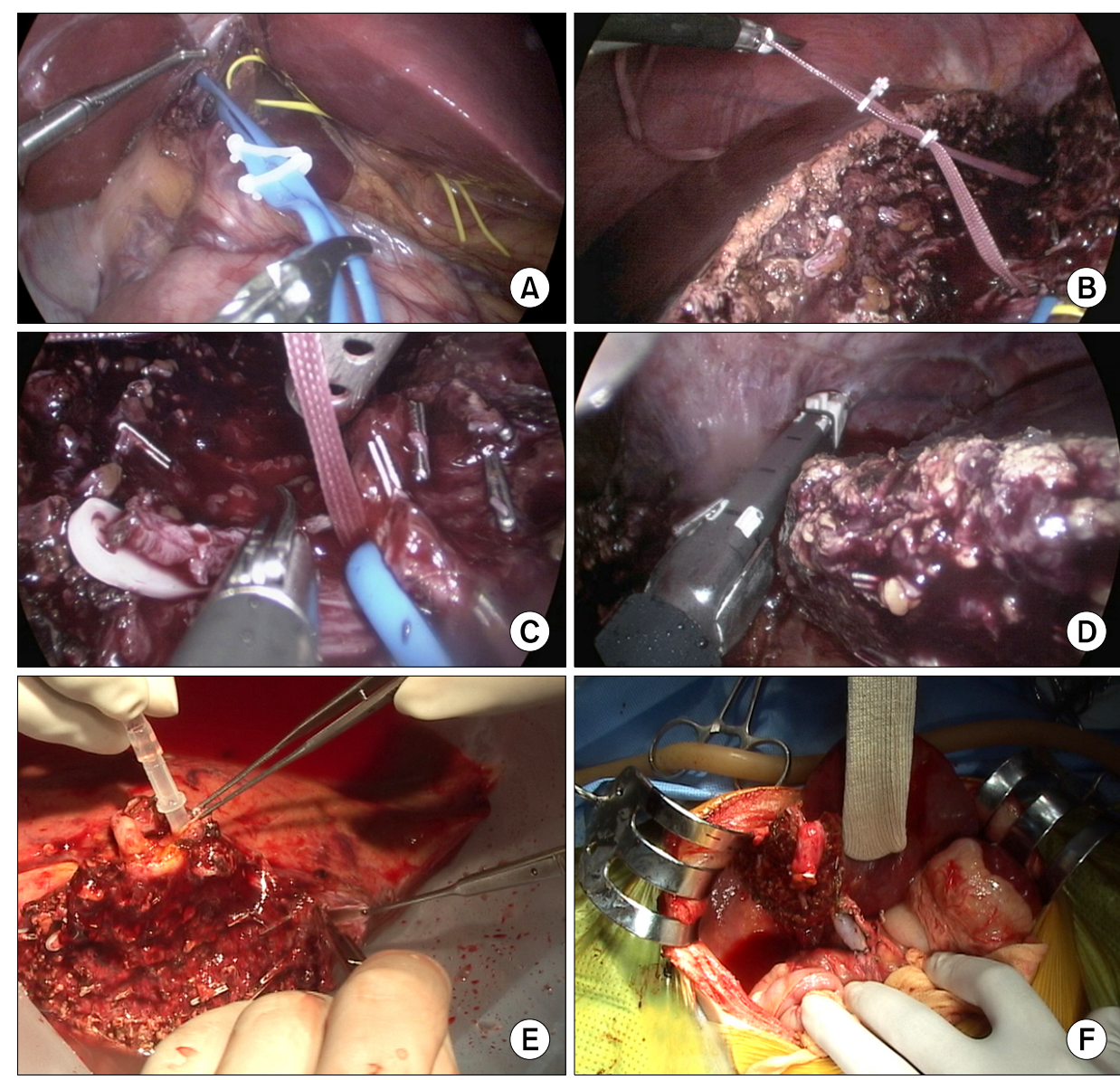

Fig. 3. Operative procedures of the laparoscopic living donor left lateral sectionectomy. (A) Left hepatic artery (a yellow tape) and portal vein (a blue tape). (B) Hanging-over maneuver. (C) $\mathrm{Cu}$ tting the left hepatic duct just above the Hem-O-Lock clip. (D) Cutting the left hepatic vein using ENDO TA $30 \mathrm{~mm}$. (E) Flushing the graft on the back table. (F) Graft implantation.

liver cut surface. Two closed-suction drains were inserted to prevent fluid collection around the liver cut surface. These procedures and results are described elsewhere. ${ }^{2}$ All surgical procedures are shown in Fig. 3.

\section{Comparison study (laparoscopy surgery vs. open surgery)}

Soubrane et al. reported that 16 successive donor underwent a laparoscopic liver resection from 2001 to 2005 . $^{3}$ They were compared with 14 other donors who underwent a standard open liver resection during a first period (1998-2004). Laparoscopic harvesting was successfully performed in 15 of 16 cases in an intention-to-treat basis. One conversion was required to ensure the quality of the laparoscopic repair of a left portal vein injury occurring during the pedicle dissection. No specific complication related to laparoscopy was observed. As compared with open liver resection, the operation time was longer in laparoscopic resection group ( $320 \pm 67$ vs. $244 \pm 55$ minutes, $p$ $<0.005)$. The blood loss was significantly lower in the
Table 1. Comparison study by Soubrane et al. ${ }^{3}$

\begin{tabular}{lccl}
\hline & $\begin{array}{c}\text { Laparoscopy } \\
\text { group }(\mathrm{n}=16)\end{array}$ & $\begin{array}{c}\text { Open group } \\
(\mathrm{n}=14)\end{array}$ & $p$-value \\
\hline $\begin{array}{l}\text { Operative time } \\
\text { (mins) }\end{array}$ & $320 \pm 67$ & $244 \pm 55$ & $p<0.005$ \\
$\begin{array}{lccl}\text { Blood loss (ml) } \\
\text { Morbidity rate }\end{array}$ & $18.7 \pm 44.2$ & $199.2 \pm 185.4$ & $p<0.005$ \\
\hline
\end{tabular}

laparoscopic liver resection group $(18.7 \pm 44.2$ vs. $199.2 \pm 185.4 \mathrm{ml}, p<0.005)$. The morbidity rate was similar in both groups $(18.7 \%$ in laparoscopic group vs. $35.7 \%$ in open group). One donor in the laparoscopic liver resection group experienced a bile leak treated by redo laparoscopy. Grafts were anatomically similar irrespective of the use of laparoscopy. The duration of hospital stay and use of self-infused morphine pump was not different between the 2 groups. In conclusion, left lateral section harvesting by laparoscopy is a safe and reproducible procedure, allowing to obtaining similar grafts as compared with laparotomy and can therefore be recommended to 
Table 2. Comparison study by Kim et al. ${ }^{2}$

\begin{tabular}{lccc}
\hline & $\begin{array}{c}\text { Laparoscopy } \\
\text { group }(\mathrm{n}=11)\end{array}$ & $\begin{array}{c}\text { Open group } \\
(\mathrm{n}=11)\end{array}$ & $p$-value \\
\hline Hospital stay (days) & $6.9 \pm 0.3$ & $9.8 \pm 0.9$ & 0.00 \\
Diet (days) & $2.1 \pm 0.3$ & $2.7 \pm 0.4$ & 0.01 \\
Operative time (mins) & $330.0 \pm 67.6$ & $305.5 \pm 28.5$ & 0.27 \\
Blood loss (ml) & $395.5 \pm 72.3$ & $463.6 \pm 77.7$ & 0.06 \\
Warm ischemic time & $6.1 \pm 1.8$ & $5.1 \pm 1.1$ & 0.07 \\
$\quad$ (mins) & & & \\
Medical costs (USD) & $5,997 \pm 1,131$ & $6,100 \pm 1,145$ & 0.83 \\
\hline
\end{tabular}

transplant centers that have previous experience in laparoscopic liver resection (Table 1).

We reported that 22 living donors including 11 underwent laparoscopic surgery and 11 underwent open surgery were enrolled (May 2008-October 2009). ${ }^{2}$ The laparoscopic liver resection group had a significantly shorter hospital stay ( $6.9 \pm 0.3$ vs. $9.8 \pm 0.9$ days, $p=0.00)$ and a shorter time to diet $(2.1 \pm 0.3$ vs. $2.7 \pm 0.4$ days, $p=0.01)$. There was no statistical difference between the laparoscopy group and the open liver resection group in operative time, blood loss, warm ischemic time and out of pocket medical costs $(330.0 \pm 67.6$ vs $305.5 \pm 28.5$ minutes; $395.5 \pm 72.3$ vs. $463.6 \pm 77.7 \mathrm{ml} ; 6.1 \pm 1.8$ vs. $5.1 \pm 1.1 \mathrm{mi}-$ nutes; $5,997 \pm 1,131$ vs. $6,100 \pm 1,145$ USD). There was no mortality in both groups. There was only one complication, wound seroma, in the open liver resection group. In conclusion, laparoscopic liver resection group is more beneficial for living donors and a safe, feasible, and reproducible procedure for living donor liver transplantation (Table 2).

\section{LAPAROSOCPY-ASSISTED LIVING DONOR HEPATECTOMY}

Although laparoscopic lateral sectionectomy, in living donors for pediatric LDLT, has been successful, a total laparoscopic right hepatectomy under pneumoperitoneum has been scarcely reported in living donors. In fact, a laparoscopic right hepatectomy is difficult to perform even in the treatment of benign tumors according to a report by Suh et al. ${ }^{4}$ This difficulty is related to 1 ) extensive mobilization of the heavy right liver that is deeply seated below the rib cage, 2) ligation of the short hepatic veins, and 3) the deep parenchymal transection needed to retain both in-flow and out-flow of the liver. Furthermore, in liv-
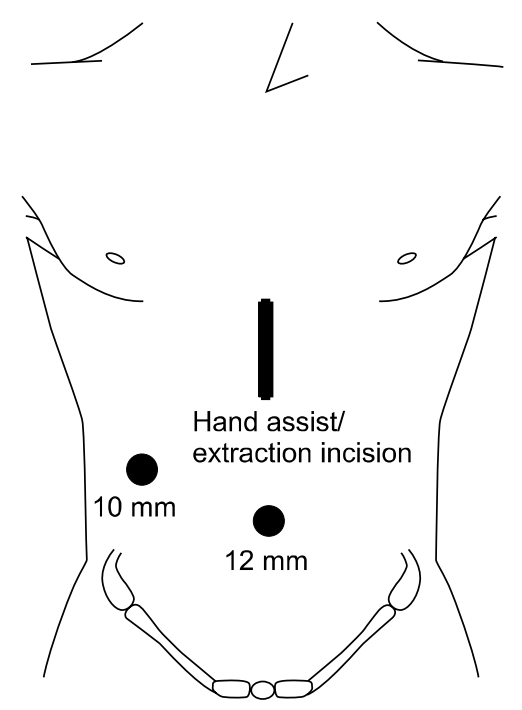

Fig. 4. Port placement during laparoscopy-assisted living donor right hepatectomy presented by Koffron et al. ${ }^{6}$

ing donors, the vascular and biliary pedicles must be distinctly divided at an adequate level without use of linear staplers or an electric coagulator; these vessels are very short and small. This is why the first minimally invasive approach to donor right hepatectomy was a mini-incision procedure. Although not frequently performed as laparoscopic left lateral sectionectomy, recently there have been some reports about the feasibility and safety of utilizing the laparoscopy-assisted approach for donor right hepatectomy. ${ }^{4-8}$

\section{Laparosopy-assisted living donor right hepa- tectomy}

Since the operative procedure for laparosopic assisted donor right hepatectomy has not been standardized, comparing with that for laparoscopic living donor left lateral sectionectomy, we introduce some procedures performed in recent reports. Koffron et al. first published a report of a living donor right hepatectomy utilizing a minimally invasive laparosopic technique. ${ }^{6}$

The donor was placed in supine position, arms adducted and urinary catheter, arterial and central venous lines were inserted under general anesthesia. Pneumoperitoneum $\left(\mathrm{CO}_{2}\right.$ at $\left.12 \mathrm{mmHg}\right)$ was established through a 12 $\mathrm{mm}$ umbilical port and the abdomen explored utilizing a $30^{\circ}, 10 \mathrm{~mm}$ laparoscope (Hopkins, Karl Storz Endoscopy, Culver City, CA, USA) for optimal optics. Once the liver was visualized one additional $10 \mathrm{~mm}$ port was placed at 
the right midclavicular line and a $5 \mathrm{~cm}$-long subxiphoid midline incision created for hand assistance during right lobe manipulation and for graft extraction (Fig. 4) using a GelPort hand port device (Applied Medical, Rancho Santa Margarita, CA, USA). This configuration enabled the surgeon, who stands on the left side of the patient, to use the hand port for graft manipulation, with simultaneous use of the midclavicular line port for dissection.

Using LigaSure $^{\mathrm{TM}}$ (Valley Lab, Boulder, CO, USA) the ligamentum teres, falciform, coronary and right triangular ligaments were divided. The hepatic bare area was completely mobilized, an then the right lobe was elevated, exposing the length of the retrohepatic inferior vena cava (IVC). The posterior vena cava ligament and short hepatic veins were divided using LigaSure separating the IVC and right lobe allowing visualization of the right hepatic vein (RHV) as it entered the IVC. An umbilical tape was passed around the RHV, the right lobe allowed to return to anatomical position and a hand-assisted cholecystectomy was performed. The right hepatic artery (RHA) and right portal vein (RPV) were isolated posteriolaterally, using the LigaSure ${ }^{\mathrm{TM}}$ with hand-assisted laparoscopy, as they entered the right lobe.

At this point, the hand port and all other laparoscopic devices were removed and, using the hand-assist/extraction incision, under direct vision, the cystic duct was cannulated in preparation for cholangiogram. Once cholangiography confirmed the biliary anatomy, the right hepatic duct was isolated, and using fluoroscopic cholangiographic guidance the right hepatic duct was then transected leaving a $4 \mathrm{~mm}$ stump with the donor hepatic confluence which was oversewn with 7-0 monofilament, non-absorbable suture. The RHA and RPV branches were further dissected under direct vision.

No inflow occlusion was applied at any point in the case. Central venous pressure was maintained at 2-4 $\mathrm{mmHg}$ in preparation for parenchymal transection. Using the hand-assist/extraction incision the liver parenchyma was divided at Cantlie's line, under direct vision, from an inferior/anterior to superior/posterior direction using the Helix Hydrojet ${ }^{\mathrm{TM}}$ (ERBE USA Incorporated Surgical Systems, Marietta, GA, USA) and the TissueLink BPS2.3 $3^{\mathrm{TM}}$ (TissueLink Medical, Dover, NH, USA). The middle hepatic vein tributaries from segments five and eight were ligated. The umbilical tape was used toward the end of the parenchymal transection to bring the transection plane closer to the surface.

Once the hemilivers were separate and the recipient operative team notified, the RHA was ligated just lateral to the common bile duct and transected sharply with scissors distally through the hand-assist incision, allowing back bleeding from the graft side. Immediately the RPV was stapled but not divided using the vascular TA device (US Surgical, Norwalk, CT, USA). The right lobe was retracted laterally and, under direct vision, the RHV was stapled and divided at the IVC using an endovascular GIA stapler (US Surgical). The RPV was then transected sharply distally to the staple line using scissors. The right lobe was decompressed by allowing back bleeding through the graft side of the RPV and the right lobe was extracted, using gentle traction, by grabbing it at the gallbladder fossa and removing it through the original hand-assist subxiphoid incision.

A completion cholangiogram was performed to confirm the integrity of the left biliary system and to rule out any leaks. A closed suction drain was placed in proximity to the remnant liver cut surface and was exteriorized through the right subcostal port site and secured to the skin with 3-0 nonabsorbable suture. The remaining port sites and the extraction incision were closed in layers. Estimated blood loss was $150 \mathrm{ml}$, the majority arising from decompression of the right lobe graft prior to extraction.

On the back table, the vascular staple lines on the RHV and RPV were excised, and the right lobe graft was flushed with Custodiol ${ }^{\mathbb{R}}$ HTK Solution (Odyssey Pharmaceuticals, East Hanover, NJ, USA) within 90s of removal.

Although not published yet in the literature, we have also performed laparoscopy-assisted living donor right hepatectomy, which was nearly the same procedure Wakabayashi et al. ${ }^{8}$ presented. The procedure was as follows: The donor was placed in the supine position, arms and legs both adducted. The operator stood by the right side of the patient while the first assistant and cameraman stood by the left side. Pneumoperitoneum $\left(\mathrm{CO}_{2}\right.$ at 12 $\mathrm{mmHg}$ ) was established and the abdomen was explored with a $30^{\circ}$ laparoscope through a $12-\mathrm{mm}$ umbilical port. One monitor each was placed on the right and left side of the donor. Three additional trocars were inserted along an imaginary right subcostal incision line. The three trocars were spaced according to the surgeon's preference. 


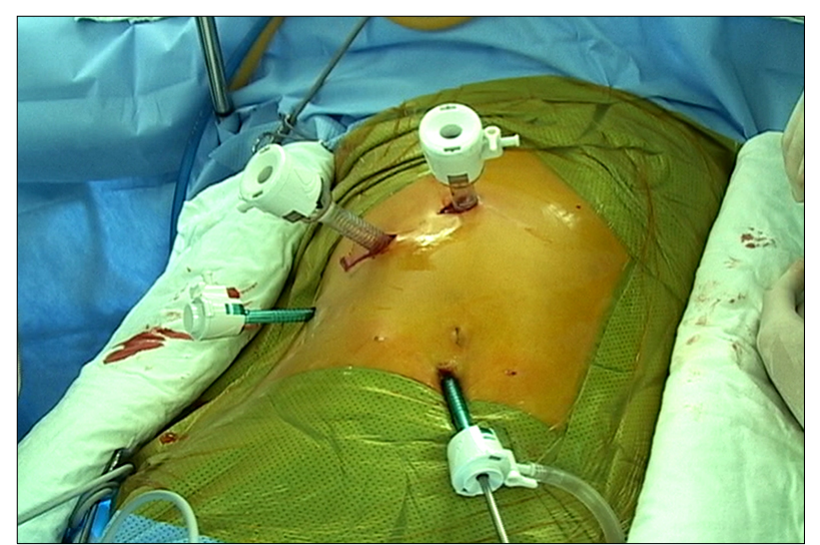

Fig. 5. Port position for laparosopy-assisted living donor right hepatectomy.

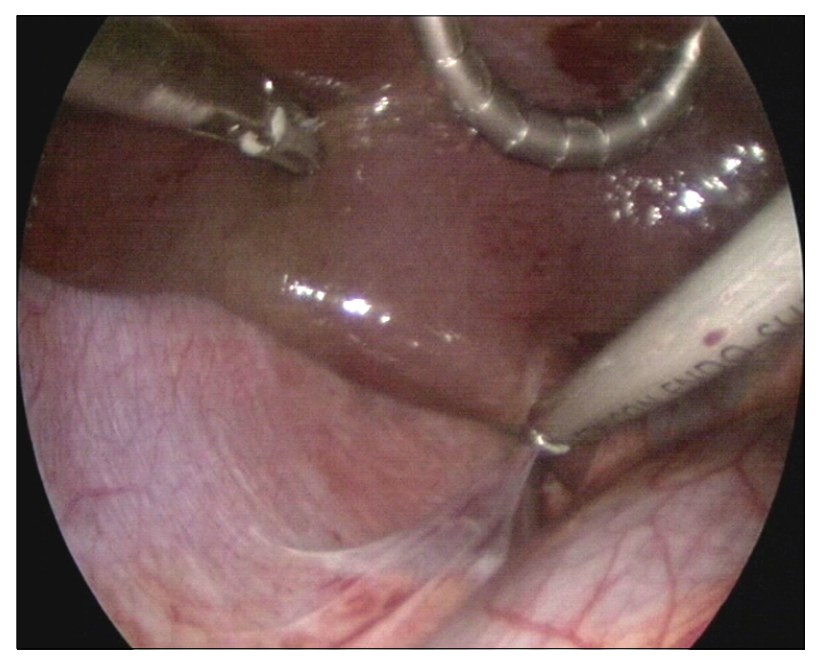

Fig. 6. Division of the right triangular and coronary ligaments with a harmonic scalpel.

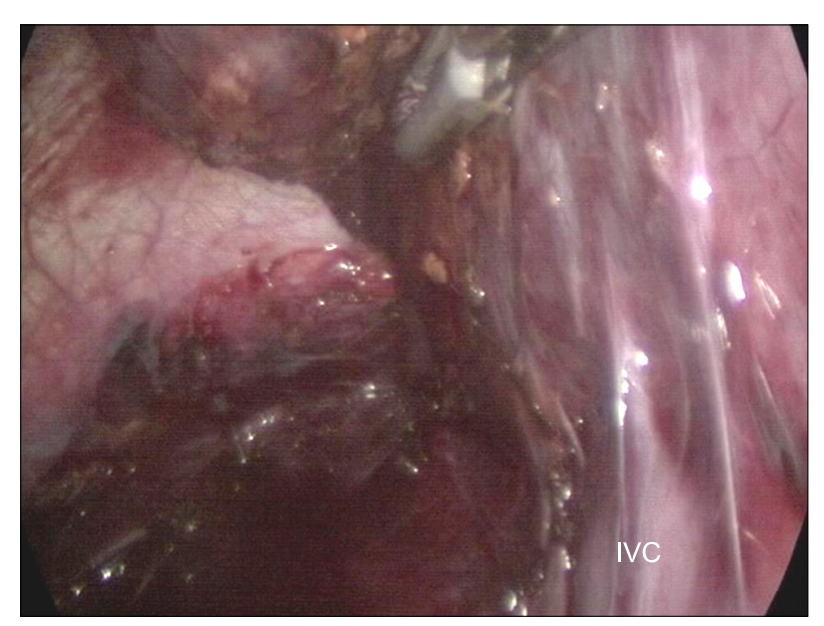

Fig. 7. Exposure of the short hepatic veins and inferior vena cava (IVC) ligament.

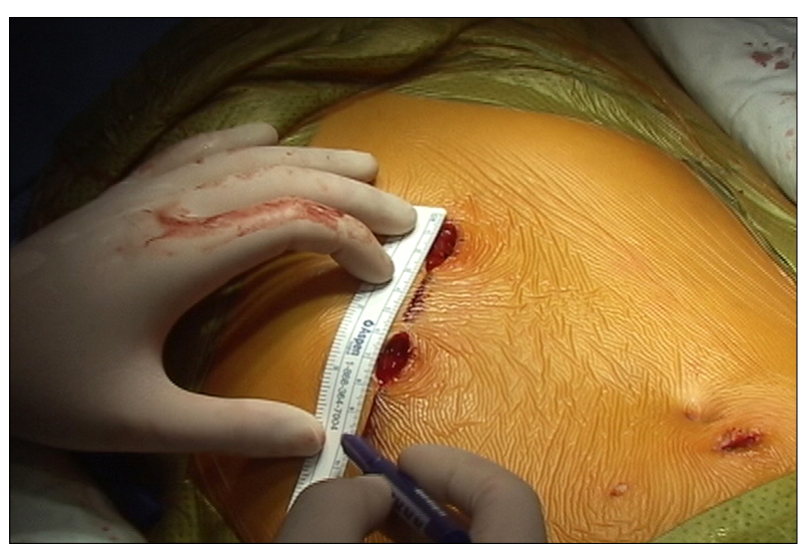

Fig. 8. $12 \mathrm{~cm}$-long subcostal incision.

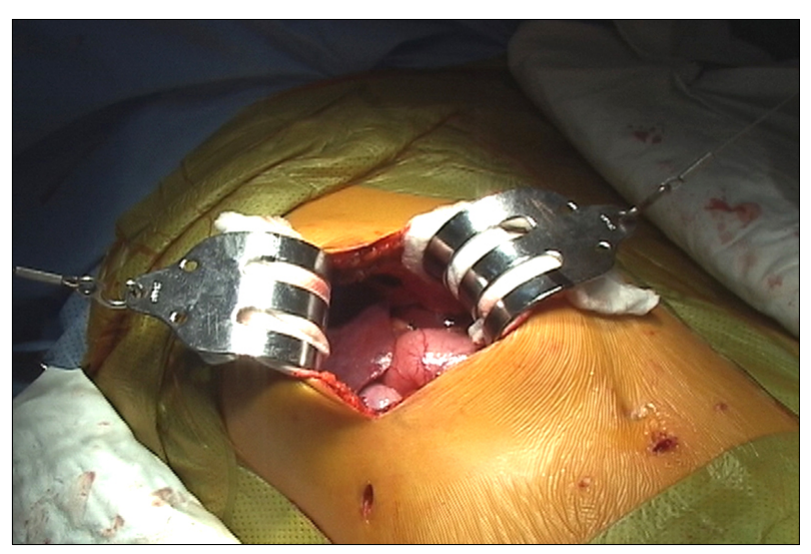

Fig. 9. Preparation for liver transection.

The middle and lateral trocars were used as primary working devices. The medial trocar was used to retract the liver medially (Fig. 5).

The liver was inspected and partially resected for a frozen biopsy to confirm the degree of steatosis. The right triangular and coronary ligaments were divided with a harmonic scalpel (Ultracision, Ethicon Endosurgery, Cincinnati, $\mathrm{OH}$ ) and monopolar electrocautery to mobilize the right lobe of the liver and expose the short hepatic veins and inferior vena cava (IVC) ligament (Fig. 6, 7). The right lobe was allowed to return to the anatomical position and at this point, the trocars and all other laparoscopic devices were removed.

The remained parts of the operation including ligation of the hepatic veins and IVC ligament, completion of hilar dissection and parenchymal transection were performed as was done for open right donor hepatectomy after a 12 cm-sized mini-laparotomy incision, which was an extension of the two medial subcostal trochar sites, was 


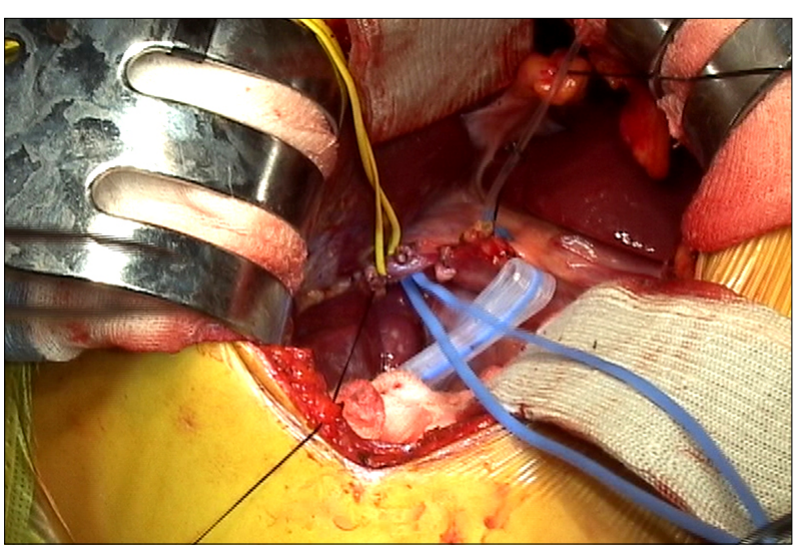

Fig. 10. Right hepatic artery (a yellow tape) and portal vein (a blue tape).

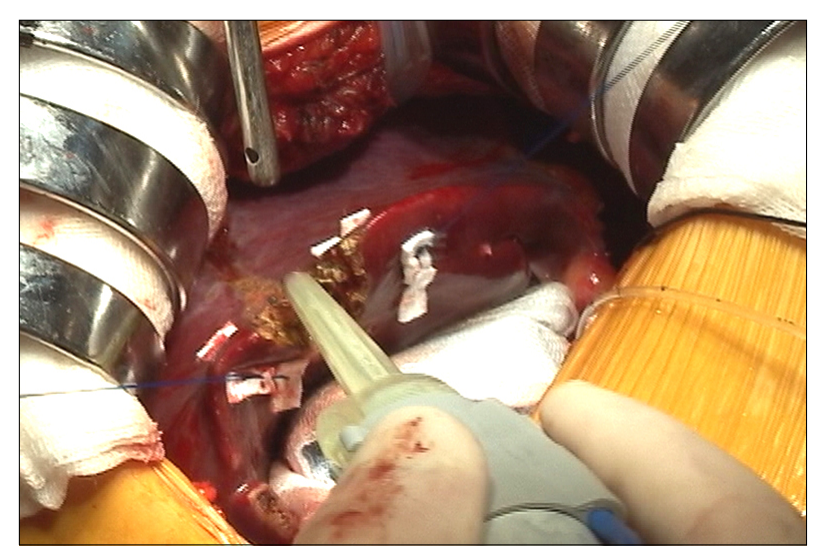

Fig. 11. Parenchymal transection with a ultrasonic aspirator (CUSA).

made (Fig. 8, 9). A silastic penrose drain was inserted between the liver and IVC for the hanging-over maneuver during parenchymal transection. After cholecystectomy was performed, using the subcostal incision, under direct vision, the cystic duct was cannulated in preparation for cholangiogram. The right hepatic artery (RHA) and right portal vein (RPV) were isolated posteriolaterally as they entered the right lobe (Fig. 10). A transection line was drawn during transient clamping of the hepatic artery and portal vein on the right side of the liver. Parenchymal transection was performed using a ultrasonic aspirator (CUSA EXcel, Valleylab Corp., Boulder, CO) without the Pringle maneuver on either side of the liver (Fig. 11). The major MHV branches ( $>5 \mathrm{~mm}$ in diameter) were isolated and divided using Hem-o-lock clips (Fig. 12). After the parenchymal dissection was completed, the right hepatic duct was isolated and divided. The bile duct stump was

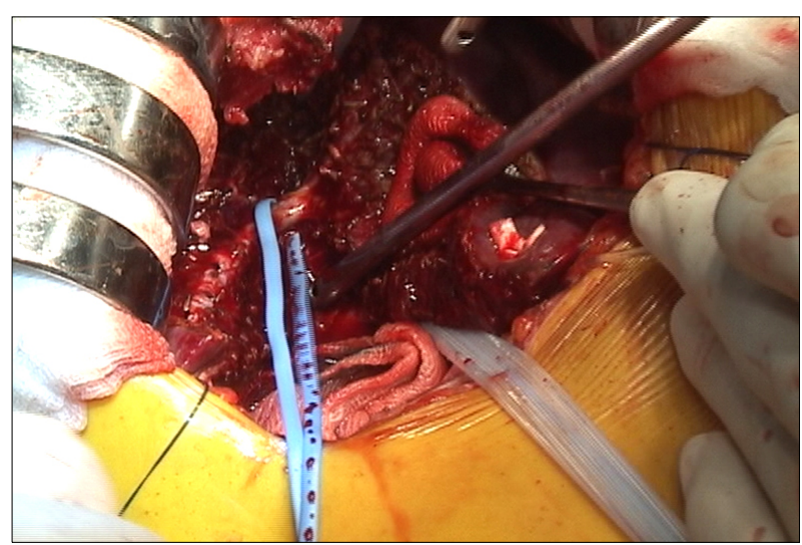

Fig. 12. No. 5 middle hepatic vein branch (a blue tape).

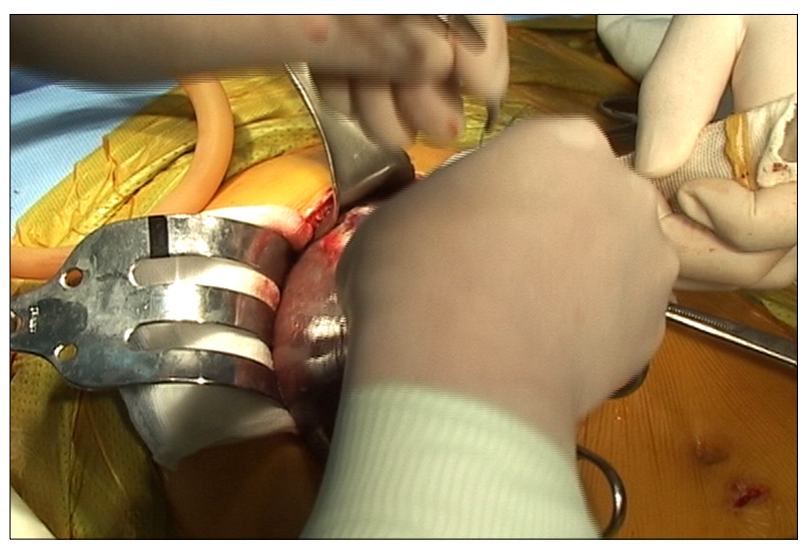

Fig. 13. Graft procurement through the mini-laparotomy site.

Table 3. Comparison study by Baker et al. ${ }^{7}$

\begin{tabular}{lccl}
\hline & $\begin{array}{c}\text { Laparoscopy-ass } \\
\text { group }\end{array}$ & Open group & $p$-value \\
\hline $\begin{array}{c}\text { Operative time } \\
\text { (mins) }\end{array}$ & $265 \pm 48$ & $316 \pm 61$ & 0.001 \\
$\begin{array}{c}\text { Blood loss (ml) } \\
\text { Hospital stay } \\
\text { (days) }\end{array}$ & $417 \pm 217$ & $550 \pm 305$ & 0.1 \\
$\begin{array}{c}\text { Hospital event } \\
\text { costs (USD) }\end{array}$ & 1.107 & 1.000 & 0.19 \\
\hline
\end{tabular}

sutured with 6-0 Prolene sutures.

After the recipient was ready to receive the graft, 5000 $\mathrm{U}$ heparin was infused and the right hepatic artery and portal vein with the right hepatic vein were divided after application of a vascular clamp. The graft was extracted through the site of the subcostal incision (Fig. 13).

After graft procurement, it was flushed on the back table with $2 \mathrm{~L}$ of $4^{\circ} \mathrm{C}$ HTK solution (Odyssey Pharmaceuti- 
cals, East Hanover, NJ, US) through the portal vein. On the back table, after removing the Hem-o-lock clips, the MHV tributaries were anastomosed to a cryopreserved deceased-donor vessel graft. The remaining liver cut surface was checked for bleeding and bile leakage. Also the remnant liver was fixed to the falciform ligament with a non-absorbable suture. Two closed-suction drains were inserted to prevent fluid collection around the liver cut surface.

\section{Comparison study (laparoscopy-assisted surgery vs. open surgery)}

Adult LDLT often requires a right hepatic lobectomy to provide sufficient graft size for the recipient. Despite open donor right hepatectomy (ODRH) being well described and characterized as a safe procedure, it is associated with complications in $38 \%$ of donors, according the largest review of living donor complications. Over the past two decades, laparoscopic surgery has been utilized for an increasing number of surgical procedures with the aim of reducing postoperative pain, recovery time, and surgical morbidity.

Although there have been numerous reports comparing laparosopic with open hepatic resection, to date, there exists only a single report comparing laparoscopy-assisted with open living donor right hepatectomy by Baker et al. ${ }^{7}$ In that report, the authors performed a retrospective, comparative analysis of 33 cases laparoscopy-assisted donor right hepatectomy (LADRH), which were performed between January 2006 and May 2008, to the most recent 33 cases of ODRH (April 2004 to July 2007) performed at their institution, evaluating donor complications, costs, and recipient outcomes. Corresponding recipient records were also reviewed. Two cases in the LADRH group were converted to open donation at the surgeon's discretion in the interest of donor safety. The outcomes for these 2 donors are reported as LADRH, according to intention-to-treat principles. LADRH was performed as described previously by Koffron et al. ${ }^{6}$

Donor demographics for LADRH and ODRH including age, gender, and body mass index (BMI) were similar. Donor complication rates were equivalent for LADRH and ODRH. Donor operative times were shorter for
LADRH (LADRH 265 minutes, ODRH 316; $p<0.001$ ) even after adjusting for BMI. Blood loss and length of stay were comparable. Additionally, total hospitalization costs were equivalent (LADRH \$1.11, ODRH \$1.00; $p=0.19$ ). Higher operative supply costs for LADRH were balanced by higher time-based operative costs for ODRH resulting in no significant differences in total operative costs. Finally, there were no differences in graft size, recipient patient or graft survival, or recipient vascular or biliary complications. Baker et al. ${ }^{7}$ concluded that LADRH can be performed as safely as ODRH with reduced operative times, a trend toward reduced blood loss, and comparable complication rates, length of stay, and hospital and surgical costs. Furthermore, LADRH provided equitable grafts for the recipient with equal patient and graft survival and complication rates (Table 3 ).

We believe that the potential benefits of a smaller incision superimposed on shorter recovery time may change the landscape for living liver donors leading to an increased willingness to donate as has been demonstrated for kidney transplantation. Further study enrolling larger amount of cases and including assessment of patient-reported health status and satisfaction, is necessary to determine if these benefits are being realized.

\section{REFERENCES}

1. Cherqui D, Soubrane O, Husson E, et al. Laparoscopic living donor hepatectomy for liver transplantation in children. Lancet 2002;359:392-396.

2. Kim KH, Jung DH, Park KM, et al. Comparison of open and laparoscopic live donor left lateral sectionectomy. $\mathrm{Br} \mathrm{J}$ Surg 2011;98:1302-1308.

3. Soubrane O, Cherqui D, Scatton O, et al. Laparoscopic left lateral sectionectomy in living donors: safety and reproducibility of the technique in a single center. Ann Surg 2006;244:815-820.

4. Suh KS, Yi NJ, Kim T, et al. Laparoscopy-assisted donor right hepatectomy using a hand port system preserving the middle hepatic vein branches. World J Surg 2009;33:526-533.

5. Troisi R, Debruyne R, Rogiers X. Laparoscopic living donor hepatectomy for pediatric liver transplantation. Acta Chir Belg 2009;109:559-562.

6. Koffron AJ, Kung R, Baker T, et al. Laparoscopic-assisted right lobe donor hepatectomy. Am J Transplant 2006;6:2522-2525.

7. Baker TB, Jay CL, Ladner DP, et al. Laparoscopy-assisted and open living donor right hepatectomy: a comparative study of outcomes. Surgery 2009;146:817-823.

8. Wakabayashi G, Nitta H, Takahara T, et al. Standardization of basic skills for laparoscopic liver surgery towards laparoscopic donor hepatectomy. J Hepatobiliary Pancreat Surg 2009;16:439-444. 